\title{
Cost Effectiveness of using Faecal Immunochemical Testing (FIT) as an Initial Diagnostic Investigation for Patients with Lower Gastrointestinal Symptoms Suggestive of Malignancy.
}

\section{Christopher Kearsey}

University of Liverpool

Catherine Graham

Warrington and Halton Teaching Hospitals NHS Foundation Trust

Harry Lobb ( $\boldsymbol{\sim}$ hslobb@gmail.com )

Bristol Royal Infirmary

Jerry Chako

University of Liverpool

Rachael Weatherburn

Royal Liverpool University Hospital

Paul Rooney

Royal Liverpool University Hospital

\section{Research Article}

Keywords: suspected colorectal malignancy, endoscopy, faecal immunochemical testing (FIT)

Posted Date: January 18th, 2021

DOl: https://doi.org/10.21203/rs.3.rs-144427/v1

License: (우 This work is licensed under a Creative Commons Attribution 4.0 International License. Read Full License 


\section{Abstract}

\section{Background}

There has been an increase in the numbers of patients presenting to primary care with suspected colorectal malignancy and subsequently an increase in demand for endoscopy. This study aims to forecast the cost of faecal immunochemical testing (FIT) compared to conventional diagnostic tests as a primary investigation for patients with symptoms suggestive of colorectal malignancy.

\section{Methods}

Retrospectively, 1950 patients with symptoms suggestive of colorectal malignancy who were referred through primary care and underwent investigations through standard endoscopic evaluation were included. These patients were used to forecast the cost of faecal immunochemical testing creating theoretical data for sensitivity and specificity. Outcome measures included: the number of investigations under current protocol; cost of current investigations; number of predicted false negatives and false positives and positive/negative predictive values using current sensitivity data for FIT; the cost forecast of using FIT as the primary investigation for colorectal malignancy.

\section{Results:}

Median age was 65 (IQR 47-82) with $43.7 \%$ male and 56.3\% female. A total of 1950 investigations were carried out with a diagnostic yield of 26 cancers ( 18 colon, 8 rectal), 138 polyps and 29 high risk adenomas (HGD +/- >10mm). In total, $£ 713,948$ was spent on the investigations. The commonest investigation was colonoscopy totalling $£ 533,169$. The total cost per cancer diagnosis was $£ 27,459$. Sensitivity $(92.1 \% \mathrm{Cl} 86.9-$ 95.3) and specificity (85.8\% $\mathrm{Cl} 78.3-90.1)$ for FIT in colorectal cancer was taken from NICE and was costed via the manufacturer(s). The projected total cost of FIT for the same population using a $\geq 4 \mu \mathrm{g}$ haemoglobin cut off was $£ 415,680$ ( $£ 15,554$ per cancer). The total cost of high-risk polyps using $\geq 4 \mu \mathrm{g}$ cut off was $£ 404,427$ (sensitivity $71.2 \% \mathrm{Cl} 60.5-87.2$, specificity $79.8 \% \mathrm{Cl} 76.1-83.7$ ) or $£ 13,945$ per polyp.

\section{Conclusions:}

FIT is a cheaper and effective alternative test with the potential to replace current expensive methods. The forecast is based on the limited data available for sensitivity/specificity in the current literature. FIT has now been commenced for symptomatic patients in the UK and therefore sensitivity may change in the future.

\section{Introduction}

Faecal Immunochemical Test (FIT) is a quick and well tolerated investigation designed to identify serious colorectal pathology. The test identifies small quantities of blood in the patient's stool (faecal occult blood $(\mathrm{FOB})$ ) using dedicated monoclonal antibodies to haemoglobin $(\mathrm{Hb})$. Most colorectal pathology with the potential to become malignant tends to bleed more than native tissue. Therefore, the presence of blood in the 
faeces potentially indicates the presence of disease in the colon and/or rectum. Those who receive a positive FIT result precede to endoscopic investigation.

Currently, the standard protocol for patients presenting with symptoms associated with the lower gastrointestinal tract is investigation with endoscopy, thereby creating a large volume of requests for these services. A colonoscopy is an expensive, invasive procedure with significant risks and patient experience is often unfavorable.

Secondary to increase in demand, endoscopy departments are struggling to meet the needs of the current referral system and it has been found many colonoscopies are being performed on large quantities of patients who are cancer free, questioning the need for change ${ }^{1}$.

NICE Guidance DG30 now recommends FIT should be used as a "straight to test" diagnostic tool for patients whose symptoms are suggestive of colorectal cancer. This paper is a cost forecast model for the use of FIT as an initial diagnostic tool prior to endoscopy or CT in symptomatic, two-week-wait (2WW) lower gastrointestinal (GI) referral patients.

\section{Materials And Methods}

Patients who presented to primary care with lower GI symptoms suggestive of colorectal cancer were urgently referred according to standardised 2WW criteria created by the Department of Health, UK and the National Institute of Health and Care Excellence (NICE). Specialist nurses who had received detailed training on the process triaged the referrals and categorised patients into different groups for investigation. All patients could qualify for more than one group. All patients who underwent investigation were sent bowel preparation by post with information about the colonoscopy procedure. Individual suitability for the test was confirmed on the day by assessment by the endoscopist on a 'case by case' basis.

Patients with normal tests were immediately discharged. Any positive findings were then reviewed by senior clinicians and it was decided at this point whether the patient a) was discharged, b) was sent for further investigations or treatment, or c) brought to clinic to discuss findings. Any patients who were deemed to have either an equivocal diagnosis or positive cancer diagnosis were referred to the local multi-disciplinary team (MDT) meeting for further management.

The data collection process was retrospectively performed on all patients who were referred under the urgent referral pathway with possible lower gastrointestinal cancer to Royal Liverpool University Hospital between March 2015 and November 2017. Demographic details and specific referral benchmarks from primary care were also documented.

From collected data, a reverse cost forecast analysis was performed using sensitivity/specificity methods by Westwood et al. ${ }^{2}$ This data was then used to estimate false positives, false negatives, the positive predictive value (PPV) and the negative predictive value (NPV) that would have likely been in our population of patients if we had used FIT as the primary investigation. These values were then used to determine the cost of FIT compared to the current protocol. 


\section{Results}

A total of 1950 patients were triaged through our service with a sex bias of, male (43.7\%) and female (56.3\%). Patient demographics included in the study are displayed in Table 1 below.

Table 1

patient demographics

\begin{tabular}{|ll|}
\hline Demographics & \\
\hline Total Number of Patients & 1950 \\
\hline Number of Males & 852 \\
\hline Number of Females & 1098 \\
\hline Median Age & 65.00 \\
\hline Interquartile Range & $(47-82)$ \\
\hline Median Age for Males & 65.00 \\
\hline Median Age for Females & 65.00 \\
\hline Average Pathway Duration & 63.01 \\
\hline SD for Pathway Duration & 45.59 \\
\hline
\end{tabular}

Median age of referral was 65 with a median local population age of 70 . As expected, the main modalities of investigation were endoscopic (colonoscopy and flexible sigmoidoscopy) and radiological (CT colonography) with some patients undergoing aditional gastroscopy due to national guidelines for iron deficiency anaemia. The distribution of these investigations are shown in Fig. 1.

Patients were coded and categorised to one or more groups depending on their symptoms. The highest number of patients were found in the $>50$ years group and with a change in bowel habit (looser stool +/more frequent stool) $(n=754)$. There were no patients who coded into the $<50$ years group with rectal bleeding and weight loss and no patients with a positive FOB screening referral. This was expected as they are investigated through a separate pathway.

The most common diagnosis was diverticular disease with a total of 474 patients. This was followed by ulcerative colitis $(n=39)$. A total of 26 cancer diagnoses were found, of which $18(69 \%)$ were colon cancer and $8(31 \%)$ were rectal cancer. There were 1323 patients with no diagnosis after investigation and were deemed normal/none-serious results and discharged. These figures are represented in Fig. 2.

A total of 1950 investigations were conducted which reported the finding of 26 cancers (approximately one cancer per 90 investigations) and 29 high-risk polyps (> $10 \mathrm{~mm}+/$ - high grade dysplasia) resulting in a $2.7 \%$ yield of significant neoplasia. Figure 3 shows the total cost of investigations that took place. These were calculated using the tariffs released by the NHS for 2018/19. Naturally, the most significant cost is colonoscopy, which had the greatest number of patients $(n=1323)$ at a single test cost of $£ 403$ ( $£ 465$ with biopsy). This was followed by CT colonography $(n=591)$ at a cost of $£ 196$ per scan. Flexible sigmoidoscopy 
$(n=252)$ cost $£ 310$ per test ( $£ 372$ with biopsy) and finally gastroscopy $(n=178)$ at $£ 341$ per test ( $£ 388$ with biopsy).

Tables 2 and 3 show the sensitivity and specificity of FIT at each of the $\mathrm{Hb}$ cut off values and the predicted false positives and false negatives modelling our population on FIT for CRC and high-risk polyps.

Table 2

- FIT sensitivities/specificities at various $\mathrm{Hb}$ cut offs and modelling data for CRC.

\begin{tabular}{|lllllllllll|}
$\begin{array}{l}\text { Hb } \\
\text { Threshold }\end{array}$ & Sensitivity & $\begin{array}{l}95 \% \\
\mathrm{Cl}\end{array}$ & Specificity & $\begin{array}{l}95 \% \\
\mathrm{Cl}\end{array}$ & $\begin{array}{l}\text { True } \\
+\end{array}$ & $\begin{array}{l}\text { True } \\
-\end{array}$ & $\begin{array}{l}\text { False } \\
+\end{array}$ & $\begin{array}{l}\text { False } \\
-\end{array}$ & $\begin{array}{l}\text { PPV } \\
(\%)\end{array}$ & $\begin{array}{l}\text { NPV } \\
(\%)\end{array}$ \\
\hline $4 \mu \mathrm{g}$ & 97.2 & $\begin{array}{l}85.5- \\
99.9\end{array}$ & 88.4 & $\begin{array}{l}58.1- \\
92.7\end{array}$ & 26 & 1895 & 258 & 0.8 & 2 & 99.9 \\
$10 \mu \mathrm{g}$ & 92.1 & $\begin{array}{l}86.9- \\
95.3\end{array}$ & 85.8 & $\begin{array}{l}78.3- \\
90.1\end{array}$ & 26 & 1895 & 314 & 2 & 7.5 & 99.8 \\
$15 \mu \mathrm{g}$ & 92.3 & $\begin{array}{l}86.6- \\
96.1\end{array}$ & 86.9 & $\begin{array}{l}85.6- \\
88.1\end{array}$ & 26 & 1895 & 286 & 2 & 8.2 & 99.8 \\
$20 \mu \mathrm{g}$ & 89.5 & $\begin{array}{l}84.9- \\
93.1\end{array}$ & 86.6 & $\begin{array}{l}85.4- \\
87.7\end{array}$ & 26 & 1895 & 293 & 3 & 8 & 99.8 \\
\hline
\end{tabular}

Table 3

- FIT sensitivities/specificities at various $\mathrm{Hb}$ cut offs and modelling data for high-risk polyps.

\begin{tabular}{|lllllllllll|}
\hline $\begin{array}{l}\mathrm{Hb} \\
\text { Threshold }\end{array}$ & Sensitivity & $\begin{array}{l}95 \% \\
\mathrm{Cl}\end{array}$ & Specificity & $\begin{array}{l}95 \% \\
\mathrm{Cl}\end{array}$ & $\begin{array}{l}\text { True } \\
+\end{array}$ & $\begin{array}{l}\text { True } \\
-\end{array}$ & $\begin{array}{l}\text { False } \\
+\end{array}$ & $\begin{array}{l}\text { False } \\
-\end{array}$ & $\begin{array}{l}\text { PPV } \\
(\%)\end{array}$ & $\begin{array}{l}\text { NPV } \\
(\%)\end{array}$ \\
\hline $4 \mu \mathrm{g}$ & 71.2 & $\begin{array}{l}60.5- \\
87.2\end{array}$ & 79.8 & $\begin{array}{l}59.7- \\
88.1\end{array}$ & 29 & 1895 & 503 & 11 & 6 & 99.2 \\
$10 \mu \mathrm{g}$ & 68.9 & $\begin{array}{l}53.2- \\
81.4\end{array}$ & 80.2 & $\begin{array}{l}76.1- \\
83.7\end{array}$ & 29 & 1895 & 468 & 13 & 7 & 99.2 \\
$15 \mu \mathrm{g}$ & 66.7 & $\begin{array}{l}50.9- \\
79.6\end{array}$ & 83.1 & $\begin{array}{l}79.2- \\
86.5\end{array}$ & 29 & 1895 & 385 & 14 & 7 & 99.3 \\
$20 \mu \mathrm{g}$ & 64.4 & $\begin{array}{l}48.7- \\
77.7\end{array}$ & 85.7 & $\begin{array}{l}81.9- \\
88.7\end{array}$ & 29 & 1895 & 316 & 16 & 8 & 99.1 \\
\hline
\end{tabular}

For the current protocol, $£ 713,948$ was spent in this study on investigations with colonoscopy costing $£ 533,169$ alone. The endoscopic/radiological evaluation for each cancer was $£ 27,459$ and $£ 24,618$ for highrisk polyps.

Depending upon manufacturer, FIT is significantly cheaper at $£ 5$ per test. We forecasted the cost of using FIT as a primary investigation on our population using $\geq 4 \mu \mathrm{g} \mathrm{Hb}$ cut off with the following calculation (1895 $x £ 5$ $+($ true positives $\times £ 465)+($ false positives $\times £ 465)+($ false negatives $\times £ 465)$ ). The total cost of FIT would have been $£ 404,427$, equating to $£ 15,554$ per cancer. The total cost of high-risk polyps using $\geq 4 \mu \mathrm{g}$ cut off level was $£ 13,945$ per polyp.

\section{Discussion}


Colorectal malignancy (CRC) is one of the top three most common cancers in the UK but is considered to be very treatable due to recent improvements in early detection ${ }^{3}$. FIT testing was originally developed as an advancement on Faecal Occult Blood testing in bowel cancer screening. It is designed to detect small amounts of blood in faeces using monoclonal antibodies to the $\mathrm{Hb}$ molecule ${ }^{4}$. Recent evidence has found with some certainty that the use of quantitative faecal immunochemical testing has substantial benefits over the FOB test in both accuracy and compliance. This posed the question of whether FIT could be used as a primary diagnostic test rather than only for screening.

With rising awareness of CRC in the population, there has been an increase in the numbers of patients presenting to primary care with symptoms suggestive of CRC. This, coupled with a change in NICE guidance, resulted in more referrals to secondary care for $2 \mathrm{WW}$ cancer referrals and so in turn escalated the demand for colonoscopies.

If the current trend continues, Bowel Cancer UK estimates that there will be an increase in demand of 10-15\% per year for diagnostic colonoscopies ${ }^{3}$. Endoscopy services are already stretched to capacity with the increasing demand and many patients breach the recommended 2WW for investigation. Secondly, endoscopic evaluation of the bowel is not without risk and carries a false negative rate of approximately $5 \% 5$. In addition, endoscopies are expensive and can be an unpleasant experience for patients.

In an attempt to streamline the referral system, initiatives have been created in some units such as nurse led colorectal telephone assessment pathways and the so called 'straight to test' pathway. These systems have been shown to reduce the time it takes for investigations to be carried out, but studies have revealed that patients may still be subject to inappropriate investigations for their symptoms, especially due to a lack of early assessment ${ }^{6}$. Thus, the identification of a cheaper, well tolerated investigation that has a robust sensitivity is of upmost importance. FIT has the potential to be used at the point of referral from primary care to guide the need for further investigations, reduce unnecessary colonoscopies and create a more costeffective system. Despite this, it is important to consider the detection and removal of polyps during colonoscopy which reduces future CRC incidence and mortality.

When looking at $\mathrm{Hb}$ cut off values in faecal immunochemical testing, increasing the Hb cut-off decreases the sensitivity, decreases the specificity of the test and increases the number of false negatives. Using FIT with an $\mathrm{Hb}$ cut off of $4 \mu \mathrm{g} \mathrm{Hb} / \mathrm{g}$ in our population, the number of false negatives would be 0.8 . Although this is low, the acceptability of this rate on a larger scale must be considered. We forecast that any missed cancers would develop further symptoms and therefore represent at a later date. These theoretical patients with more advance disease have their own cost implications and more importantly the ethical implications associated with a missed cancer diagnosis. Robust safety netting advice for patients using FIT would be absolutely necessary.

Patients receiving negative FIT test results could be reassured they are unlikely to have cancer and discharged. Other possible outcomes of a negative FIT could be watch and wait, onward referral to colorectal outpatient clinic or repeat faecal immunochemical testing, particularly if the patient's symptoms persist. It is vital that FIT results should not be viewed in isolation and clinical judgement remains of paramount 
importance. Results for high-risk polyps shows a reduced sensitivity/specificity and a higher false negative rate of 11 at the $4 \mu \mathrm{g} \mathrm{Hb} / \mathrm{g}$ cut off and these would also have the possibility of missed malignancy.

Using a $\mathrm{Hb}$ cut-off of $4 \mu \mathrm{g} \mathrm{Hb} / \mathrm{g}$ determined 258 false positives. This can be explained as FIT detects haemoglobin associated with a variety of other pathology such as inflammatory bowel disease or high-risk polyps. Data from other studies suggested that up to $28.9 \%$ of patients with an initial false positive result from a FIT were eventually diagnosed with some form of serious bowel disease. In our forecast, the patient who received a positive result would undergo colonoscopy as per the protocol and therefore patient disruption would be minimal.

Overall, the results of this study are positive and reveal a highly sensitive and specific test that could be used as a primary investigation for $2 \mathrm{WW}$ patients to facilitate the saving of colonoscopy resources. The cost of endoscopy to NHS England in 2014 was approximately $£ 178.4$ million and it was found that approximately $40 \%$ of tests were normal ${ }^{8}$ which is comparable to our study. This study shows the cost of diagnosing colorectal cancer and high-risk polyps via endoscopy is significantly higher than the forecasted cost of FIT at $£ 27,459$ and $£ 24,618$ vs $£ 15,554$ and $£ 13,945$ respectively. This is a potential cost reduction of $43 \%$ over 2 years $(£ 309,521)$.

\section{Conclusion}

FIT is a cheaper alternative diagnostic test with the potential to replace current methods with similar sensitivity.

\section{Declarations}

\section{Ethics approval:}

All protocols were approved by Institute of Translational Medicine, University of Liverpool and the Department of Colorectal Surgery, St Helens and Knowsley Teaching Hospitals NHS Trust. All methods were performed in accordance with the institutional guidelines and regulations and informed consent was not required as Royal Liverpool University Hospital ethics committee states that anonymised, non-interventional, retrospective studies that adhere to NHS consent policy do not need formal consent and therefore the committee approves this study.

\section{Consent for publication:}

not applicable.

\section{Availability and data materials:}

The datasets used and/or analysed during the current study are available from the corresponding author on reasonable request. 


\section{Completing interests:}

The authors declare that they have no competing interests.

\section{Funding:}

University of Liverpool.

\section{Authors contributions:}

the article was written by $\mathrm{CK}, \mathrm{CG}, \mathrm{HSL}$ and JC and reviewed by RW and PSR.

\section{Acknowledgements:}

not applicable.

\section{$\mathrm{MeSH} /$ key terms}

Colorectal Neoplasms

Models, Econometric

Referral and Consultation

Diagnostic Tests, Routine

\section{References}

1. Alpha Laboratories. About Faecal Immunochemical Testing [internet] UK and Eire. Alpha Laboratories (2019) https://www.faecal-immunochemical-test.co.uk/about-faecal-immunochemical-testing

2. Westwood $\mathrm{M}$ et al. Faecal immunochemical tests (FIT) can help to rule out colorectal cancer in patients presenting in primary care with lower abdominal symptoms: a systematic review conducted to inform new NICE DG30 diagnostic guidance. BMC Medicine 2017 15:189

3. Bowel Cancer UK . Improving capacity, saving lives: endoscopy in the UK.London: Bowel Cancer UK; 2012. p. 10

4. Laish, I., Blechman, I., Feingelernt, H. and Konikoff, F. (2015). Yield of second surveillance colonoscopy to predict adenomas with high-risk characteristics. Digestive and Liver Disease, 47(9), pp.805-810.

5. Fraser, C. (2019). Faecal immunochemical tests for haemoglobin (FIT) in the assessment of patients with lower abdominal symptoms: current controversies. Gastroenterología y Hepatología, 42(4), pp.263270. 
6. Mowat, C., Digby, J., Strachan, J., Wilson, R., Carey, F., Fraser, C. and Steele, R. (2015). Faecal haemoglobin and faecal calprotectin as indicators of bowel disease in patients presenting to primary care with bowel symptoms. Gut, 65(9), pp.1463-1469.

7. McDonald, P., Digby, J., Innes, C., Strachan, J., Carey, F., Steele, R. and Fraser, C. (2013). Low faecal haemoglobin concentration potentially rules out significant colorectal disease. Colorectal Disease, 15(3), pp.e151-e159.

8. Omata F, Shintani A, Isozaki M, Masuda K, Fujita Y, Fukui T. Diagnostic performance of quantitative fecal immunochemical test and multivariate prediction model for colorectal neoplasms in asymptomatic individuals. Eur J Gastroenterol Hepatol 2011; 23: 1036-41

\section{Figures}

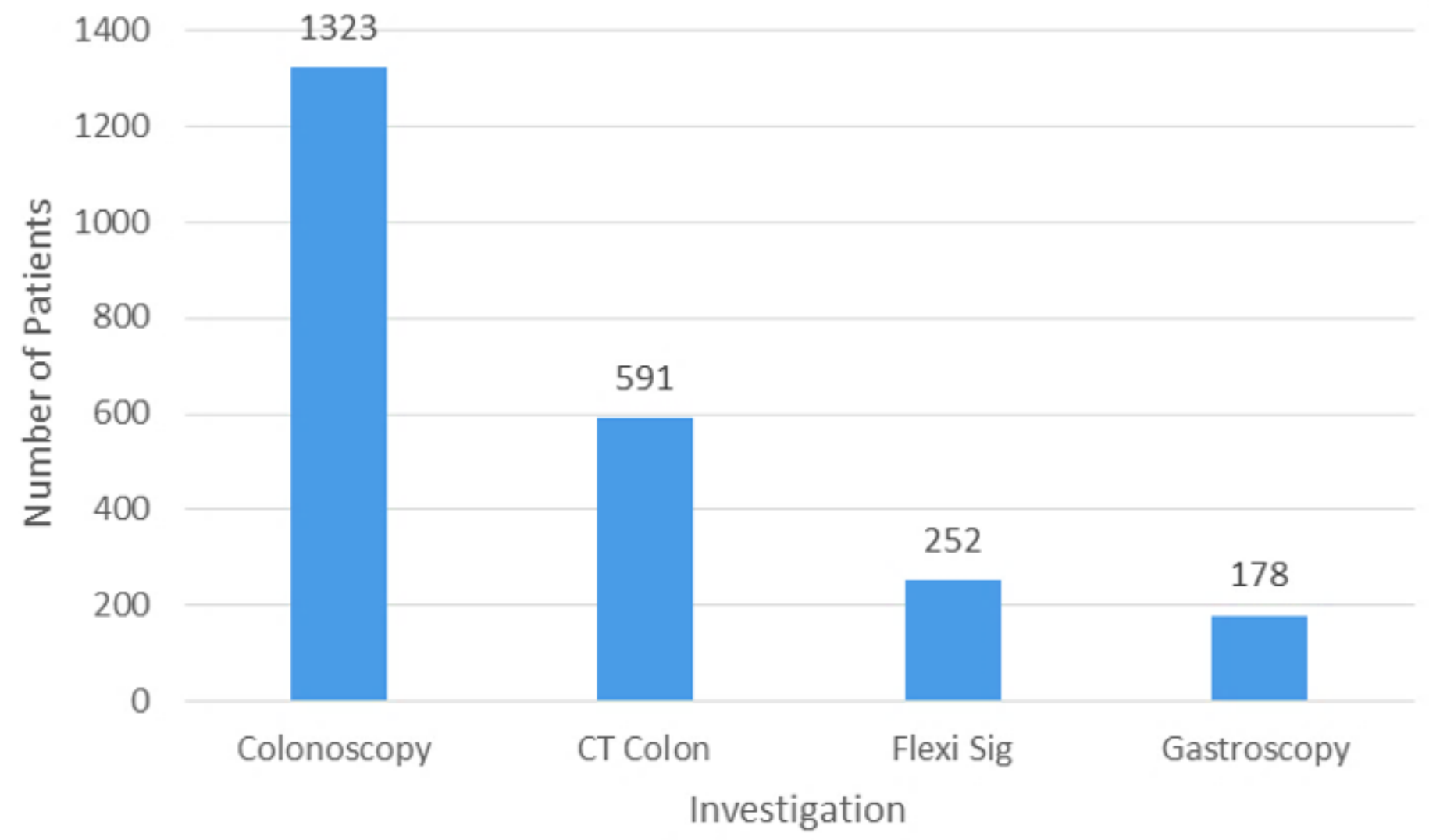

\section{Figure 1}

The distribution of investigations as per the current protocol. 


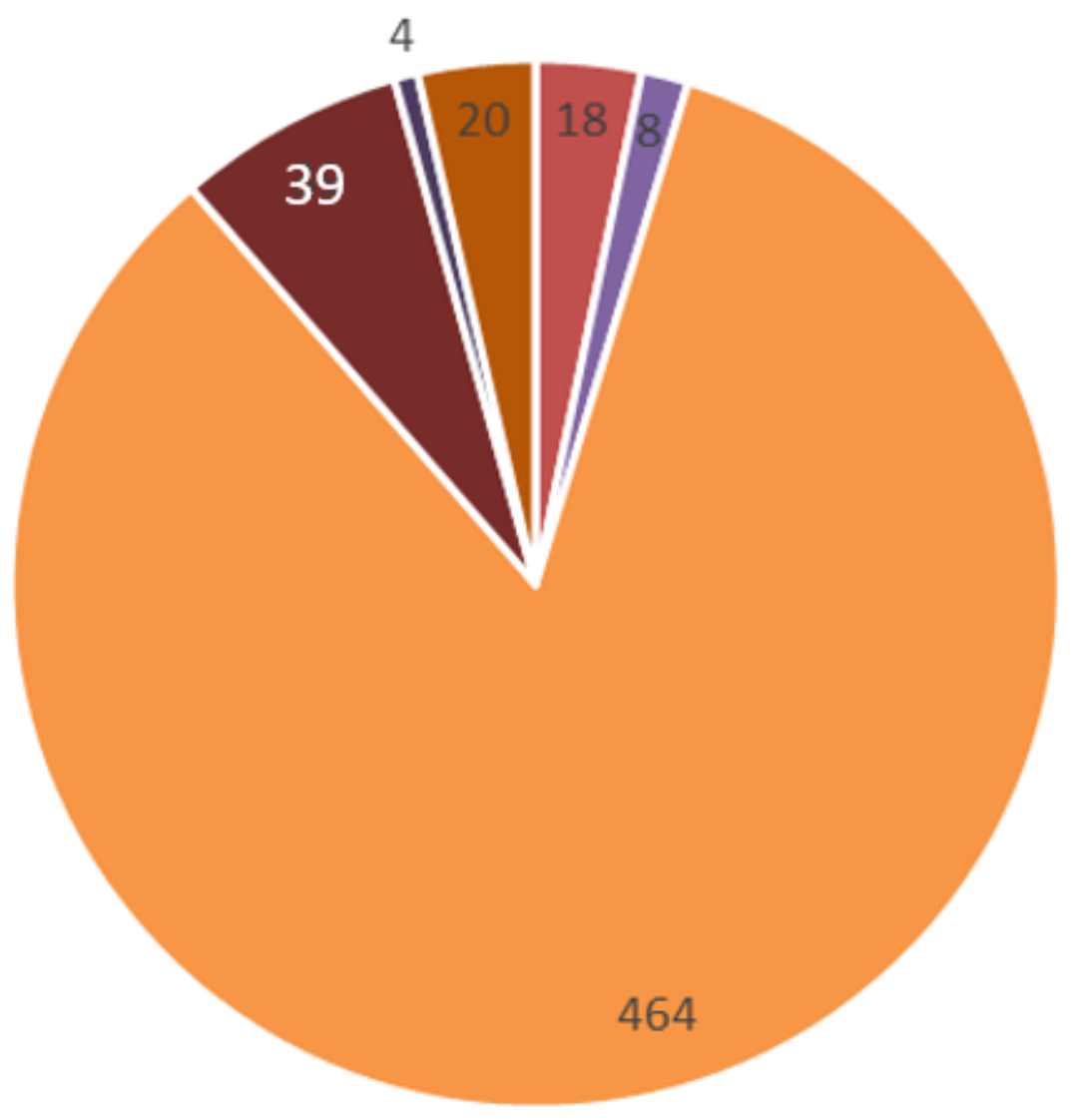
- Colon Cancer
- Rectal Cancer
- Diverticular Disease
- Ulcerative Colitis
- Crohn's Disease
- Microscopic Colitis

Figure 2

Distribution of diagnosis. 


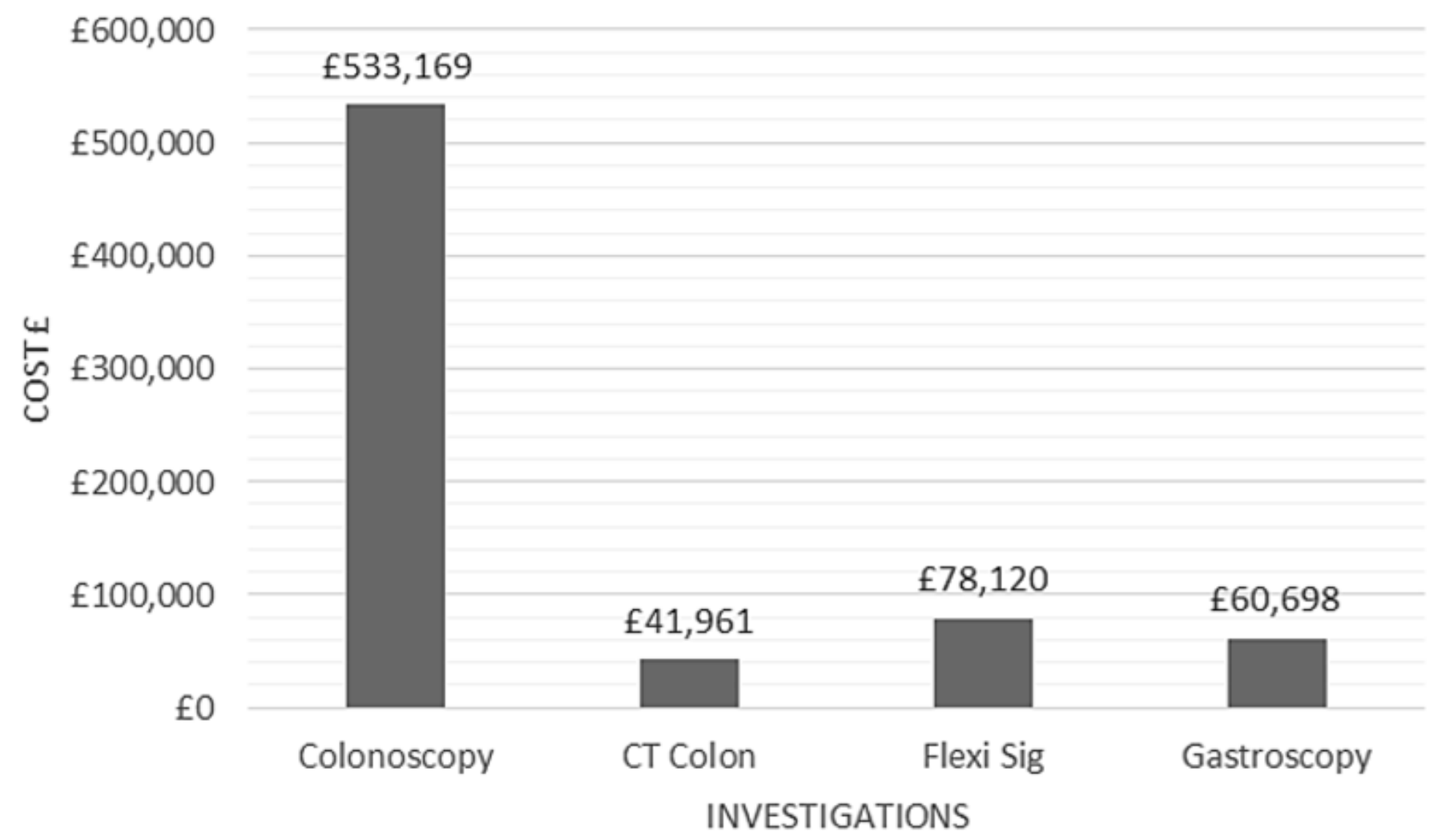

Figure 3

Cost breakdown of the investigations in the current protocol 